\title{
Improvement of a Capillary Electrophoresis-Chemiluminescence Detection System for Using a Polyacrylamide-Coated Capillary
}

\author{
Kazuhiko Tsukagoshi, *† Takahiro Kimura,* Toshinari FuJI,* Riichiro NaKaJIMA,* \\ and Akihiro ARA *** \\ *Department of Chemical Engineering and Materials Science, Faculty of Engineering, Doshisha University, \\ Kyotanabe, Kyoto 610-0321, Japan \\ **HPLC Business Department, Analytical Instruments Division, Shimadzu Corporation, \\ 1 Nishinokyo-Kuwabaracho, Nakagyo, Kyoto 604-8511, Japan
}

(Received September 7, 2000; Accepted November 2, 2000)

During the past decade, capillary electrophoresis (CE) has been shown to be a powerful and efficient analytical separation technique. CE features high resolution, relatively short analysis time, and low operational cost compared with high-performance liquid chromatography (HPLC). One of the major areas in animated research for $\mathrm{CE}$ is the development of sensitive detection methods. On-column optical detection modes, such as UV absorption and fluorescence detection, are the most commonly used on account of the extremely small sample zone and the small capillary dimensions in CE.

Chemiluminescence (CL) has been verified to be a useful detection method in both flow-injection analysis and HPLC. $\mathrm{CL}$ is also expected to be an ideal detection method for CE. Recently, the applicability of CL detection in CE has been succeessfully demonstrated. ${ }^{2-5}$ We reported on a CL detection of proteins separated by CE. ${ }^{5}$ The bis(2,4,6-trichlorophenyl)oxalate(TCPO) $-\mathrm{H}_{2} \mathrm{O}_{2}$ system was used together with fluorescent compounds.

On the other hand, various types of capillaries, including coated and gel capillaries, ${ }^{6-9}$ are used in order to improve or alter the separation selectivity in CE. However, as far as we know, the CE-CL detection methods have so far been applied for only ordinary untreated fused-silica capillaries. Therefore, although the CE-CL detection methods have shown high sensitivity, they were not always satisfactory for the separation ability in CE. There must be several problems in using any modified capillaries in the CE-CL detection methods.

We newly developed the CE-CL detection system using a polyacrylamide-coated capillary through several technical improvements. The previous system ${ }^{5}$ using an untreated fusedsilica capillary was mainly improved concerning the attachment of a flow line of an aqueous buffer solution and a mixing filter in order to provide the conductivity required for electrophoresis. The improvements led to a stable and constant electric current in the system using a coated capillary. A mixture sample of lysozyme, cytochrome $c$, and ribonuclease $a$ labeled with fluorescamine, which could not be separated by an untreated fused-silica capillary, was separated and detected.

In the development of the CE-CL detection methods having various separation modes, the interface between the $\mathrm{CE}$ and the CL detection becomes important. The results and information

† To whom correspondence should be addressed. obtained by this study must give a clue for the development of real high-performance CE-CL detection methods.

\section{Experimental}

Reagents

All of the reagents used were of commercially available special grade. Ion-exchanged water was distilled for use. Bovine serum albumin (BSA), lysozyme (Lyso), cytochrome $c$ (Cyto), and ribonuclease $a$ (Ribo) were purchased from Sigma Co. Bis(2,4,6-trichlorophenyl)oxalate (TCPO) and fluorescamine (FR) were received from Wako Pure Chemical Industries and Fluka Chemie AG, respectively. Acrylamide, methacryloxypropyltrimethoxysilane, and tetramethylethylenediamine were, respectively, purchased from Fluka Chemie AG, Shinetu Chemical Industries, and Wako Pure Chemical Industries.

\section{Preparation of a polyacrylamide-coated capillary ${ }^{10}$}

A new capillary tube of $75 \mu \mathrm{m}$ i.d. (Polymicro Technologies) was treated with $1.0 \mathrm{~mol} \mathrm{dm}^{-3}$ sodium hydroxide for $30 \mathrm{~min}$ and washed with distilled water and then acetonitrile. After a solution containing $4 \mathrm{~cm}^{3}$ of acetonitrile, $16 \mu \mathrm{l}$ of methacryloxypropyltrimethoxysilane, and $32 \mu \mathrm{l}$ of acetic acid was passed through the capillary for $30 \mathrm{~min}$, the capillary was left standing for $90 \mathrm{~min}$, and washed with acetonitrile. Acrylamide $\left(30 \mathrm{mg}\right.$ ) was dissolved in $1 \mathrm{~cm}^{3}$ of distilled water. To the solution, were added $5 \mu \mathrm{l}$ of tetramethylethylenediamine and $5 \mu \mathrm{l}$ of $10 \%$ ammonium peroxodisulfate solution. The solution was passed through the capillary and left for $1 \mathrm{~h}$. Finally, the capillary was washed with distilled water for $1 \mathrm{~h}$.

\section{Apparatus and procedures}

An outline of the present apparatus is shown in Fig. 1. A migration buffer solution $\left(10 \mathrm{mmol} \mathrm{dm}^{-3}\right.$ borate buffer, $\mathrm{pH} 9.0$ or $10 \mathrm{mmol} \mathrm{dm}^{-3}$ phosphate buffer, $\mathrm{pH}$ 3.0) was filtered through a filter (Millipore Millex-GSSLGS0250s) and degassed for use. A coated capillary tube ( $70 \mathrm{~cm}$ length) was filled with the buffer solution in advance. The CL reagent $\left(1 \mathrm{mmol} \mathrm{dm}{ }^{-3}\right.$ TCPO acetonitrile solution of $50 \mathrm{~cm}^{3}+30 \mathrm{wt} \% \mathrm{H}_{2} \mathrm{O}_{2}$ aqueous solution of $288 \mu \mathrm{l}$ ) was treated by a degasser (SD-8023, Online Degasser, TOSOH) and fed at a rate of $20 \mu \mathrm{min}^{-1}$ by a pump 


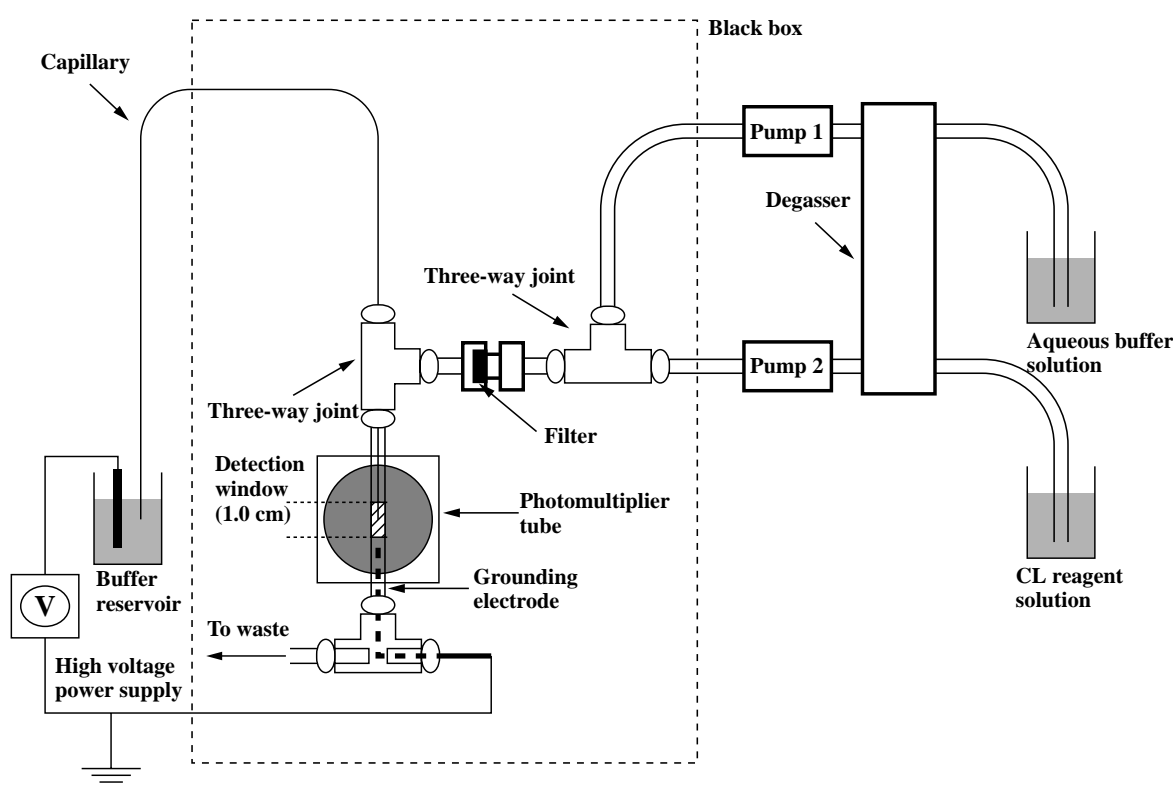

Fig. 1 Schematic diagrams of the CE-CL detection system using a polyacrylamide-coated capillary.

(P-500, Pharmacia Fine Chemicals). An aqueous buffer solution (10 mmol dm $\mathrm{m}^{-3}$ borate buffer, $\mathrm{pH}$ 9.0) was also treated by a degasser and fed at a rate of $5 \mu \mathrm{min}^{-1}$ by a pump (LC-9A, Shimadzu Co.). The CL reagent and the aqueous buffer solution were mixed just in front of the mixing filter by a threeway joint and well mixed through a filter.

A sample solution was prepared by vigorously mixing a protein solution $(450 \mu \mathrm{l})$ and a $1 \times 10^{-2} \mathrm{~mol} \mathrm{dm}^{-3} \mathrm{FR}$ acetone solution $(100 \mu \mathrm{l})$ by a vortex mixer. The aqueous buffer solution was fed and then a high voltage was applied. Subsequently, the CL reagent solution was fed until a definite electric current was observed. Then, only the voltage was removed, and the sample solution was introduced into a capillary tube for $30 \mathrm{~s}$ from $25 \mathrm{~cm}$ height by siphoning. After introducing the sample solution, a voltage of $0-15 \mathrm{kV}$ was gradually applied for $60 \mathrm{~s}$. Monitorning was started just after the voltage reached $15 \mathrm{kV}$.

A $400 \mu \mathrm{m}$ i.d. Teflon tube, which was covered with black Tygon-tape and had a $10 \mathrm{~mm}$ detection length in front of a photomultiplier (R-464, Hamamatsu Photonics Co., LTD.), was used as a detection cell. The mixture of the CL reagent and the aqueous buffer solution were mixed with the eluate at the tip of the capillary tube. The resulting CL was detected by a photomultiplier, measured by a photon counter (C1230, Hamamastu Photonics Co., LTD.), and treated by an integrator (Chromatopac C-R6A, Shimadzu Co.).

\section{Results and Discussion}

\section{Comparison of the coated and none-coated capillaries}

A frequent problem associated with $\mathrm{CE}$ using an untreated fused-silica (none-coated) capillary is the adsorption of solutes on the capillary wall. In order to obtain efficient and reproducible separations, the interactions between the solutes and the capillary wall need to be suppressed or at least tightly controlled. Chemically modifying the surface of the capillary has been developed to reduce and eliminate the ability of the solute to interact with the wall of the capillary. For example, fused-silica capillaries were modified with polyacrylamide, ${ }^{6}$ polyethylene glycol, ${ }^{7,8}$ polyethylsiloxane chains, ${ }^{7,8}$ and so on. In particular, modified capillaries were useful for the separation of proteins. Actually, a comparison of a polyacrylamide-coated and a none-coated capillaries was made for the protein separation by means of UV detection in this study. The CE system using a none-coated capillary could not separate a mixture sample of Lyso, Cyto, and Ribo, while the system using the coated capillary could separate it.

\section{Problems of the previous $C E-C L$ detection system}

When a polyacrylamide-coated capillary was applied to a CECL detection system which was previously reported by us, ${ }^{5}$ an electric current did not pass through the capillary. The electroosmotic flow was negligible in the coated capillary and an acetonitrile solvent dissolving CL reagent was mixed with an eluate at the tip of the capillary. The distance from the tip of the capillary to the electrode in the outlet reservior was about $8 \mathrm{~cm}$. The zone was almost filled up with acetnitrile; there were few electrolytes. This must have been the reason why a current did not occur in the previous system.

In order to provide conductivity between the tip of capillary and the electrode, an aqueous buffer solution was, in advance, added to the CL reagent solution. However, since TCPO is easily decomposed in an aqueous solution, the CL intensity quickly decreased when using a CL reagent solution including an aqueous buffer.

Improvement of the CE-CL detection system for a polyacrylamide-coated capillary

The several improvements were carried out in order to apply a polyacrylamide-coated capillary to a CE-CL detection. First, an aqueous buffer solution containing electrolytes was fed with another flow path and mixed with the CL reagent solution by a three-way joint. At the beginning, an effective electric current value was observed. However, the value decreased with increasing time; finally, it became zero after about $10 \mathrm{~min}$. Then, a mixing filter was equipped on the line in order to sufficiently mix the aqueous buffer solution and the CL reagent 
solution (acetonitrile solvent). After these modifications, a stable and constant electric current occurred in the system.

Next, a grounding electrode was directly inserted into the Teflon tube (detection cell) using a three-way joint. In the previous apparatus, an electrode was immersed in the outlet reservoir, so that the distance between the electrode and the tip of capillary would be about $8 \mathrm{~cm}$. The distance between them in the present apparatus became very short (about $1 \mathrm{~cm}$ ). This improvement must also be effective for maintaining a stable and constant electric current.

\section{Separation and detection of model proteins}

As a preliminary experiment, BSA labeled with FR was subjected to the present CE-CL detection system using a polyacrylamide-coated capillary. It migrated in the capillary and was detected. However, the peak on the electropherogram showed poor reproducibility. BSA has a relatively large molecular weight and a hydrophobic interaction between polyacrylamide chains and BSA must be strong. Lysozyme, Cyto, and Ribo, which have relatively small molecular weights compared with BSA, were used as model samples. Their molecular weights and isoelectropoints are as follows: Lyso (14000, 11.0), Cyto (11700, 10.1), and Ribo (13700, 9.5) (cf. BSA $(66300,4.8))$. These isoelectropoints must be somewhat different from those for labeled-proteins, but would be useful for considering their electrophoretic behaviors.

A mixture of the three proteins was examind using a migration buffer of $\mathrm{pH}$ 9.0. In both UV and CL detections, Lyso and Cyto migrated and were detected; Cyto migrated faster than Lyso, while Ribo was not detected within $1 \mathrm{~h}$. These peaks were identified by the retention time of each sample. They were worn by a positive charge under an alkaline condition. However, since the isoelectronic point of Ribo is near to the $\mathrm{pH}$ of the separation condition, the amount of positive charge must be relatively small. Their migration behaviors seem to be reasonable when thinking about their isoelectropoints together with the separation condition.

Under the alkaline condition, a mixture sample of three proteins could not be separated. Therefore, the sample was examined using a migration buffer of $\mathrm{pH} 3.0$ (the $\mathrm{pH}$ for $\mathrm{CL}$ reaction at the tip of the capillary would be maintained at about pH 9 with an excess of the aqueous buffer solution fed by the pump), because the condtion which is far from isoelectropoint of proteins must lead to good experimental results. Under an acidic condition, a mixture containing Lyso, Cyto, and Ribo was separated. The electropherogram obtained by CL detection is shown in Fig. 2. The relationship between the concentration of Lyso and the CL intensity was examined: $6 \times 10^{-7}-6 \times 10^{-6} \mathrm{~mol}$ $\mathrm{dm}^{-3}$ of Lyso could be determined with a detection limit of $6 \times$ $10^{-7} \mathrm{~mol} \mathrm{dm}^{-3}(S / N=2)$. The CL detection was about 3 and 20 times as sensitive as fluorescence (ex. $390 \mathrm{~nm}$, em. $475 \mathrm{~nm}$ ) and UV $(215 \mathrm{~nm})$ detections, respectively. The relative standard deviations of the retention time and the CL intensity for Lyso were within $5 \%$.

Bis[2-(3,6,9-trioxadecanyloxycarbonyl)-4-nitrophenyl]oxalate (TDPO) is also well known to be a peroxyoxalate CL reagent and, although TDPO is expensive, can provide a better CL performance than TCPO. Actually, TDPO gave an improvement with regard to the detection limit of Lyso.

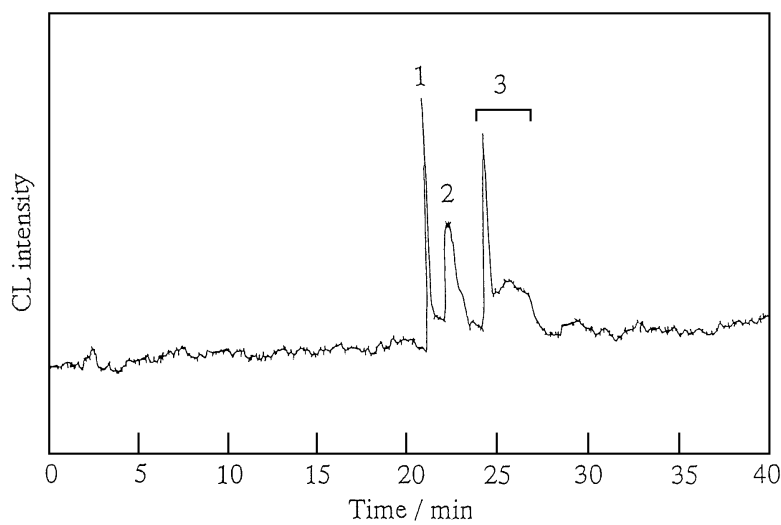

Fig. 2 Electropherograms of a mixture sample of FR-labeled Cyto (1), Lyso (2), and Ribo (3) with CL detection using a polyacrylamide-coated capillary under an acidic condition ( $\mathrm{pH} 3.0)$. Conditions: capillary, $70 \mathrm{~cm}$ of $75 \mu \mathrm{m}$ i.d. fused silica; applied voltage, $15 \mathrm{kV}$; migration buffer, $10 \mathrm{mmol} \mathrm{dm}^{-3}$ phosphate buffer (pH 3.0); and sample, $1 \times 10^{-6} \mathrm{~mol} \mathrm{dm}^{-3}$.

Lysozyme could be detected with a detection limit of $6 \times 10^{-8}$ mol dm${ }^{-3}(S / N=2)$ by using TDPO instead of TCPO.

\section{Acknowledgements}

This work was supported by a grant to RCAST at Doshisha University from the Ministry of Education, Japan. It was also supported in part by a Grant-in-Aid for Scientific Research (C) from the Ministry of Education, Science, Sports and Culture. The authors acknowledge financial support for this research by Doshisha University's Research Promotion Fund.

\section{References}

1. T. Hara and K. Tsukagoshi, Anal. Sci., 1990, 6, 797, and references cited therein.

2. R. Dadoo, A. G. Seto, L. A. Colon, and R. N. Zare, Anal. Chem., 1994, 66, 303.

3. S.-Y. Liao and C.-W. Whang, J. Chromatogr. A, 1996, 736, 247.

4. M. A. Ruberto and M. L. Grayeski, Anal. Chem., 1992, 64, 2758.

5. K. Tsukagoshi, A. Tanaka, R. Nakajima, and T. Hara, Anal. Sci., 1996, 12, 525.

6. A. Wainright, J. Microcol. Sep., 1990, 2, 166.

7. S. Terabe, H. Utsumi, K. Otsuka, T. Ando, T. Inomata, S. Kuze, and Y. Hanaoka, J. High Res. Chromatogr., 1986, 9 , 666.

8. J. A. Lux, H. Yin, and G. Schomburg, J. High Res. Chromatogr., 1990, 13, 145.

9. A. S. Cohen and B. L. Karger, J. Chromatogr., 1987, 397, 409.

10. S. Hjerten, J. Chromatogr., 1985, 347, 191. 